J Pediatr. 2013 October ; 163(4): 955-960.e1. doi:10.1016/j.jpeds.2013.04.053.

\title{
Comparative Effectiveness of Three Surfactant Preparations in Premature Infants
}

\author{
Andrea Trembath, MD, MPH ${ }^{1,{ }^{*}}$, Christoph P. Hornik, MD, MPH ${ }^{2}$, Reese Clark, MD ${ }^{3}$, P. Brian \\ Smith, MD, MPH, $\mathrm{MHS}^{2}$, Julie Daniels, $\mathrm{PhD}, \mathrm{MPH}^{4}$, and Matthew Laughon, MD, $\mathrm{MPH}^{5}$ on \\ behalf of the Best Pharmaceuticals for Children Act - Pediatric Trials Network \\ ${ }^{1}$ Department of Pediatrics, Rainbow Babies and Children's Hospital, Case Western Reserve \\ University, Cleveland, $\mathrm{OH}$ \\ ${ }^{2}$ Department of Pediatrics and Duke Clinical Research Institute, Duke University Medical Center, \\ Durham, NC \\ ${ }^{3}$ Pediatrix Center for Research and Education, Sunrise, FL, and Pediatrix Medical Group, \\ Greenville Memorial Hospital, Greenville, SC \\ ${ }^{4}$ Department of Epidemiology, University of North Carolina, Chapel Hill, NC \\ ${ }^{5}$ Department of Pediatrics, University of North Carolina, Chapel Hill, NC
}

\begin{abstract}
Objective-To compare effectiveness of three surfactant preparations (beractant, calfactant, and poractant alpha) in premature infants for preventing three outcomes: (1) air leak syndromes; (2) death; and (3) bronchopulmonary dysplasia (BPD) or death (composite outcomes).
\end{abstract}

Study design-We conducted a comparative effectiveness study of premature infants admitted to 322 neonatal intensive care units in the U.S. from 2005-2010 who were treated with beractant, calfactant, or poractant alfa. We compared the incidence of air leak syndromes, death, and bronchopulmonary dysplasia (BPD) or death, adjusting for gestational age, antenatal steroids, discharge year, and small-for-gestational-age status.

Results-51,282 infants received surfactant; $40 \%$ received beractant, $30 \%$ calfactant, and 30\% poractant alfa. Median birth weight was $1435 \mathrm{~g}$ (interquartile range 966-2065); median gestational age was 30 weeks (27-33). On adjusted analysis, we observed a similar risk of air leak syndromes (calfactant vs. beractant odds ratio [OR] $=1.17$ [95\% confidence interval: $0.95,1.43$ ]; calfactant vs. poractant $\mathrm{OR}=1.23[0.98,1.56]$; beractant vs. poractant $\mathrm{OR}=1.06[0.87,1.29]$ ), death (calfactant vs. beractant $\mathrm{OR}=1.14[0.93,1.39]$; calfactant vs. poractant $\mathrm{OR}=0.98[0.78,1.23]$; beractant vs. poractant $\mathrm{OR}=0.86[0.72,1.04])$, and $\mathrm{BPD}$ or death (calfactant vs. beractant $\mathrm{OR}=1.08[0.93,1.26]$; calfactant vs. poractant $\mathrm{OR}=1.19[1.00,1.41]$; beractant vs. poractant $\mathrm{OR}=1.10[0.96,1.27])$.

(C) 2013 Mosby, Inc. All rights reserved.

Corresponding Author: P. Brian Smith, MD, MPH, MHS, Duke Clinical Research Institute, Box 17969, Durham, NC 27715; P: 919-668-8951; F: 919-668-7058; brian.smith@duke.edu.

*A list of members of the Best Pharmaceuticals for Children Act- Pediatric Trials Network is available at www.jpeds.com (Appendix).

The other authors declare no conflicts of interest.

Publisher's Disclaimer: This is a PDF file of an unedited manuscript that has been accepted for publication. As a service to our customers we are providing this early version of the manuscript. The manuscript will undergo copyediting, typesetting, and review of the resulting proof before it is published in its final citable form. Please note that during the production process errors may be discovered which could affect the content, and all legal disclaimers that apply to the journal pertain. 
Conclusions-Beractant, calfactant, and poractant alfa demonstrated similar effectiveness in prevention of air leak syndromes, death, and BPD or death in premature infants when adjusted for site. Previously described differences in mortality between surfactants likely do not represent true differences in effectiveness but may relate to site variation in outcomes.

\section{Keywords}

beractant; calfactant; poractant alfa; premature infants; respiratory distress syndrome

Respiratory distress syndrome (RDS) causes significant morbidity and mortality in premature infants. Exogenous surfactant replacement therapy for the treatment of RDS in premature infants decreases severe RDS, pulmonary air leak syndromes, and death. ${ }^{1}$ Three animal-derived surfactants are commercially available in the United States-beractant (Survanta $\left.{ }^{\circledR}\right)$, calfactant $\left(\operatorname{Infasurf}{ }^{\circledR}\right)$, and poractant alfa $\left(\right.$ Curosurf $\left.^{\circledR}\right)$. All three preparations are approved by the Food and Drug Administration (FDA) for use in infants to treat RDS. Over the last 10 years, the use of surfactant in the United States has changed little, from 16\% to $19 \%$ among infants admitted to a neonatal intensive care unit (NICU). ${ }^{2}$ However, the relative use of specific surfactant preparations has changed significantly. The use of beractant has decreased from $95 \%$ to $42 \%$ of all surfactant administrations, and the use of calfactant and poractant alpha has increased from $5 \%$ to $27 \%$ and $0 \%$ to $29 \%$, respectively. ${ }^{2}$

Understanding the comparative effectiveness of surfactant preparations is important for reducing neonatal morbidity and mortality. However, randomized trials comparing the efficacy of surfactant preparations have often demonstrated equivocal results or were terminated early due to lack of enrollment. ${ }^{3-6}$ No completed prospective studies directly comparing the efficacy of the three surfactants within the same trial exist. Due largely to cost and recruitment issues, further study through a head-to-head randomized trial of surfactant therapy is unlikely, thus retrospective comparative effectiveness analyses or metaanalyses are justified to determine the differences, if any, between surfactant preparations.

A recent retrospective cohort study suggested that poractant alfa was associated with a reduced risk for in-hospital mortality when compared with calfactant and beractant (though comparison with beractant was not statistically significant). ${ }^{7}$ Other experts have argued that this conclusion is problematic as it is based on a retrospective study of an administrative data set that is not a part of a daily documentation system. ${ }^{8}$ In addition, a significant portion of the sample was not included in final models for analysis. A meta-analysis examining randomized trials of porcine versus bovine surfactants in RDS also suggests that infants treated with poractant alfa have a decreased risk of death as compared with those treated with beractant. ${ }^{9}$ The trials included in the meta-analysis, which date from 1995-2005, represent a small number of patients and may not be representative of current clinical practice or effectiveness. We compared the effectiveness of beractant, calfactant, and poractant alfa for preventing three outcomes: (1) air leak syndromes; (2) death; and (3) bronchopulmonary dysplasia (BPD) or death (composite outcome).

\section{METHODS}

We conducted a comparative effectiveness study using an administrative database of infants discharged from 322 NICUs managed by the Pediatrix Medical Group from January 1, 2005, to December 31, 2010. Clinicians who provide direct care to infants in these NICUs generate data on a daily basis for the purposes of creating progress notes and medical billing. Daily notes are stored in an electronic database along with administered medications and diagnoses. From the daily notes, data are extracted, de-identified (in compliance with the Health Insurance Portability and Accountability Act of 1996), and consolidated into the 
Pediatrix BabySteps Clinical Data Warehouse. This study was approved by the Duke University Institutional Review Board and Western Institutional Review Board.

We included all inborn infants with a gestational age (GA) $<37$ completed weeks who were cared for at a single NICU and received beractant, calfactant, or poractant alfa. We excluded infants admitted to NICUs that administered surfactant to $<50$ infants over the study period, as well as infants who received $>1$ surfactant preparation (Figure).

Air leak syndrome was defined as a diagnosis of pneumothorax or pulmonary interstitial emphysema following the first exposure to surfactant. Infants <32 weeks GA were classified as having BPD if they received supplemental oxygen or respiratory support (nasal canula, continuous positive airway pressure, or mechanical ventilation) continuously from a corrected GA of $360 / 7$ weeks to 36 6/7 weeks (designated as the test period). Infants $\geq 32$ weeks GA at birth were classified as having BPD if they received supplemental oxygen or respiratory support (nasal canula, continuous positive airway pressure, or mechanical ventilation) continuously from a postnatal age of 28-34 days. The receipt of continuous respiratory support or supplemental oxygen was required to more clearly define infants with BPD as compared with those with a transient need for oxygen. Infants on room air without any respiratory support during the respective test period were classified as not having BPD. Infants discharged on room air prior to the test period and not receiving respiratory support on the day of discharge were classified as not having BPD. Those who died before the test period were classified as not having BPD. The outcome of BPD was left as missing if the infant was discharged prior to the test period while receiving supplemental oxygen or respiratory support. The composite outcome of BPD or death was defined as the diagnosis of $\mathrm{BPD}$ and or all-cause in-hospital mortality.

\section{Statistical Analyses}

We used summary statistics to describe subjects according to the surfactant administered. We compared categorical and continuous variables across the three surfactant types using the chi-square tests of association and non-parametric Kruskal-Wallis tests, respectively. To account for the correlated structure of our data within NICUs, we fit unconditional logistic regression models as well as mixed models with random and fixed effects for NICUs.

We compared outcomes between infants who received beractant, calfactant, or poractant alfa. Using prior knowledge of potential confounders, we included GA, birth weight, smallfor-gestational-age (SGA) status, antenatal steroid exposure, sex, race, and discharge year. We used a backward elimination method to determine if our a priori covariates should remain in the model and compared models with the full model containing all covariates using likelihood ratio tests with a significance cut point of $<0.1$. The final variables included in the model were GA, antenatal steroid exposure, SGA status, and discharge year.

For each outcome, we used the Hausman specification test to evaluate the correlation between a NICU-specific effect and the included covariates. Given that the Hausman test rejected the null hypothesis $(P<0.001)$ for each outcome modeled, we concluded that there was correlation between unobserved NICU-specific effects and the variables included in our models. As a result, we opted to use conditional fixed effects logistic regression models for our primary analyses. Conditioning on NICU addressed the heterogeneity of baseline risk of outcomes in each NICU and allowed us to best estimate the treatment effect. The results of unconditional logistic regression and random effects models were also included to compare with estimates from prior studies. Effect measure modification was evaluated by including interaction terms with GA and surfactant and by conducting likelihood ratio tests using a significance cut-point of $<0.1$. No interaction terms were found to be significant. All 
analyses were conducted using STATA statistical software version 12 (College Station, TX), and a $P$-value $<0.05$ was considered statistically significant.

\section{RESULTS}

We identified 51,282 infants with a median birth weight of $1435 \mathrm{~g}$ (interquartile range [IQR]: 966-2065) and a median gestational age of 30 weeks (27-33) (Table I). Overall, 40\% of infants $(n=20,383)$ were treated with beractant, $30 \%(n=15,748)$ with calfactant, and $30 \%$ $(\mathrm{n}=15,151)$ with poractant alfa. During this time period, the use of beractant and calfactant decreased and the use of poractant alfa increased.

Infants treated with poractant alfa were more mature and larger (median GA 31 weeks [IQR: 28-34], birth weight $1590 \mathrm{~g}$ [1050-2220]) when compared with those treated with beractant (GA 30 weeks [27-33], birth weight $1390 \mathrm{~g}$ [940-2020]) or calfactant (GA 30 weeks [2733], birth weight $1360 \mathrm{~g}$ [930-1960]). A higher proportion of infants treated with calfactant were born to mothers who were treated with antenatal steroids (Table I).

Air leak occurred in 3450 infants (7\% overall; $8 \%$ beractant, $7 \%$ calfactant, $5 \%$ poractant alfa). Death occurred in 4576 infants (9\% overall; $10 \%$ beractant, $9 \%$ calfactant, $7 \%$ poractant alfa). A total of 12,164 infants (22\% overall; $27 \%$ beractant, $25 \%$ calfactant, $20 \%$ poractant alfa) had a diagnosis of BPD or death (composite) (Table II). Three percent of infants $(\mathrm{n}=1514)$ were missing data for determining the outcome of BPD, and 3\% $(\mathrm{n}=1506)$ were missing data for the outcome of BPD or death.

\section{Regression Models}

Fixed Effects Models-For the outcome of air leak syndromes, we observed no significant differences among the three surfactants (calfactant vs. beractant odds ratio [OR] $=1.17$ [95\% confidence interval: $0.95,1.43]$, calfactant vs. poractant $\mathrm{OR}=1.23[0.98,1.56]$, and beractant vs. poractant $\mathrm{OR}=1.06[0.87,1.29])$ using a fixed effects model (Table III). For the outcome of death alone, the fixed effects model showed no significant differences among the three surfactants (calfactant vs. beractant $\mathrm{OR}=1.14[0.93,1.39]$, calfactant vs. poractant $\mathrm{OR}=0.98[0.78,1.23]$, beractant vs. poractant $\mathrm{OR}=0.86[0.72,1.04])$.

For the combined outcome of BPD or death, the fixed effects model showed no significant differences among the three surfactants (calfactant vs. beractant $\mathrm{OR}=1.08[0.93,1.26]$, calfactant vs. poractant $\mathrm{OR}=1.19[1.00,1.41]$, and beractant vs. poractant $\mathrm{OR}=1.10[0.96$, $1.27])$.

Simple Logistic Regression and Random Effects Models-For the outcome of air leak syndromes and the composite outcome of BPD or death, there was a statistical difference between poractant versus calfactant or beractant in the simple logistic regression models (air leak syndromes: calfactant vs. poractant $\mathrm{OR}=1.25$ [1.13, 1.40], and beractant vs. poractant $\mathrm{OR}=1.47[1.35,1.61]$ for $\mathrm{BPD}$ or death: calfactant vs. poractant $\mathrm{OR}=1.04[0.95$, $1.13]$, and beractant vs. poractant $\mathrm{OR}=1.35[1.26,1.43])$. However, for the outcome of death no difference was noted between calfactant and poractant (calfactant vs. poractant $\mathrm{OR}=1.04$ $[0.95,1.13]$. The random effects models showed similar statistical results to the fixed effects models with the exception of air leak syndromes (calfactant vs. poractant $\mathrm{OR}=1.23[1.04$, $1.44]$ and beractant vs. poractant $\mathrm{OR}=1.31[1.13,1.51])$.

\section{DISCUSSION}

In this large cohort of infants, we found that beractant, calfactant, and poractant alfa had similar relative effectiveness at preventing air leak syndromes, death, and BPD or death in 
premature infants. These results are important as they indicate that there may be no clear advantage of one surfactant over another based on important outcome measures. Given our sample size of approximately 15,000 infants treated with poractant and 15,000 infants treated with beractant, we had $80 \%$ power to demonstrate a $1.4 \%$ absolute difference for the combined outcome of BPD or death.

Air leak syndromes are associated with short- and long-term morbidities including hypotension, hypoxia, and intraventricular hemorrhage. Surfactant administration decreases air leak syndromes such as pneumothorax and pulmonary interstitial enphysema when compared with placebo. ${ }^{5,8-12}$ In this study, we found three surfactant preparations to be similar to each other in preventing air leak syndromes. Overall, the incidence of air leak has decreased significantly in the last several decades, likely due to a number of factors including non-invasive ventilation, improvements in the technology of mechanical ventilation, and surfactant therapy. ${ }^{13-16}$ The incidence of pneumothorax among infants $<30$ weeks GA in this study was similar to the incidence across the Vermont Oxford Network (0-8.6\% from 2005-2010), supporting the assumption that these data are representative of national estimates. ${ }^{17}$

BPD is the most common serious pulmonary outcome in premature infants and is inversely proportional to GA and birth weight. ${ }^{18}$ The incidence of BPD has not decreased despite advances in respiratory care, in part due to increased survival of the lowest GA infants who are at highest risk of BPD. ${ }^{14}$ Because surfactant therapy decreases the severity of RDS, it was believed that surfactant might also lower the incidence of BPD. In addition, it seemed possible that differences in surfactant preparations, such as surfactant proteins, might affect the incidence of BPD. However, previous studies have not demonstrated a significant difference in the risk of BPD with surfactant use or between different surfactant preparations. ${ }^{3,4,19}$ Likewise, we observed no differences in the incidence of BPD or death among surfactant preparations.

Prior to the availability of surfactant, mortality was a common outcome for extremely premature infants. ${ }^{20}$ During our study period, mortality decreased among all but the lowest gestational ages, with infants born at $<24$ weeks gestation. This is consistent with 2010 data from the Vermont Oxford Network, which showed that mortality among infants with birth weights between $500 \mathrm{~g}$ and $1500 \mathrm{~g}$ was at $65 \%$ in those $<23$ weeks GA. ${ }^{17}$

Surfactant therapy was originally developed to decrease the severity of RDS in extremely and moderately preterm infants. FDA labeling for all three surfactants is based on studies that focused on infants $<30$ weeks GA and birth weights $<1250 \mathrm{~g}$ or those with evidence of significant RDS. ${ }^{5,19,21}$ However, nearly half of the infants in our cohort were either moderate- (GA between 31 0/7 weeks and 33 6/7 weeks) or late-preterm infants (GA between 34 0/7 weeks and 36 6/7 weeks). Consistent with reports from the few prior studies, our results suggest that a substantial portion of surfactant is used off-label and that current practices are not evidence-based. As the number of preterm deliveries continues to increase, of which late-preterm infants comprise the largest fraction, the role of surfactant in this population needs to be closely evaluated.

The comparative effectiveness of surfactants has become a controversial topic. ${ }^{8,22-24}$ In the study by Ramanathan et al, they conclude that "poractant alfa treatment for RDS was associated with a significantly reduced likelihood of death when compared with calfactant and a trend toward reduced mortality when compared with beractant." were 8276 patients who met the selection criteria, yet were excluded due to unreported, missing, or invalid entries for one or more of the variables: sex, race, APR-DRG, gestational age or birth weight; the exclusion of these left 14,173 patients for use in the revised 
regression models." 8 Our results, based on a larger and less selected data sample, do not support the conclusions offered by Ramanathan et al.

Site variation has been clearly linked to unexplained differences in outcomes such as BPD and death in other studies. For example, the risk of BPD ranged from 7\% to $48 \%$ among the Neonatal Research Network centers in a randomized controlled trial of benchmarking to reduce BPD in infants $<1250 \mathrm{~g}$ birth weight. ${ }^{25}$ This variation was not explained by differences in birth weight, GA, race, frequency of prenatal steroid use, or incidence of RDS. Therefore, models that do not adequately account for site variation may produce estimates that are difficult to interpret.

Differences between estimates of effectiveness among surfactant preparations in prior studies and this study may be partially attributable to definitions and statistical methods, including use of different modeling strategies. Modeling, in general, is used to evaluate the association between an outcome of interest, such as death, and a main predictor of interest, such as surfactant preparation, while controlling for other covariates. In simple logistic regression, observations are assumed to be independent from each other. In cases where subjects are clustered by center, this assumption may not be valid. Center-level effects may influence associations between the predictor of interest and outcome. ${ }^{26}$ Random or fixed effects models measure change within a group — for example, an individual center-and are often used to account for these center-specific effects. By measuring change within a center across multiple infants, these models can control for a number of potential omitted variables unique to each center. Random effects models assume that center-specific effects are uncorrelated with the other independent variables of the model, and fixed effects do not require this assumption be met. This is an advantage of fixed effects models in certain circumstances, as they remove potential bias that could result from the correlation between site-specific effects and the independent variables. In our study, we believe that certain sitespecific effects may have been correlated with the choice of surfactant, making a fixed effects modeling strategy more appropriate. This assumption was supported by the results of the Hausman test. We therefore chose the fixed effects model to provide a more conservative estimate of the true association between surfactant and outcome. The results from this more conservative modeling strategy were significantly different from those using simple logistic regression, which likely overestimated the association between surfactant type and outcomes. Our study is limited by lack of randomization, and we may not have accounted for all known and unknown confounders.

In recent years, the need for comparative effectiveness research has been fueled by the emergence of new pharmaceuticals in the marketplace, as well as a push for cost containment in medication expenditures. However, an important purpose for comparative effectiveness research is also to assist decision-making by clinicians and purchasers to improve the delivery of care. ${ }^{27}$ Prior studies have demonstrated the efficacy and cost effectiveness of surfactant as compared with placebo in the setting of randomized controlled trials; however, few have considered the effectiveness of surfactant preparations compared with one another. Surfactant is an effective therapy, and further studies that would compare all three surfactant preparations to placebo are unethical. Thus, comparative effectiveness is one of the few methods available to understand how these products are performing in clinical practice. ${ }^{27}$

In summary, we found no significant differences in the outcomes of air leak syndromes, death, and BPD or death between infants treated with beractant, calfactant, and poractant alfa. Also, nearly half of infants treated with surfactant were moderate- or late-preterm infants, representing a significant amount of off-label use of this medication. Previously described differences in mortality between surfactants likely do not represent true 
differences in effectiveness but are accounted for by unmeasured site variation in outcomes. Therefore, the decision regarding which surfactant preparation to use should be based on factors other than effectiveness.

\section{Acknowledgments}

Supported by the American Recovery and Reinvestment Act (DHHS-1R18AE000028-01 to P.B.S.). M.L. receives salary support for research in pediatric and neonatal clinical pharmacology from the US government (HHSN267200700051C, PI Benjamin, under the Best Pharmaceuticals for Children Act) and National Institutes of Child Health and Human Development (NICHD; 1K23HL092225-01). P.B.S. receives salary support for research from the National Institutes of Health, US Department of Health and Human Services (HHSN267200700051C), and NICHD (1K23HD060040-01). R.C. is an employee of the Pediatrix Center for Research and Education.

\section{ABBREVIATIONS}

$\begin{array}{ll}\text { BPD } & \text { bronchopulmonary dysplasia } \\ \text { FDA } & \text { Food and Drug Administration } \\ \text { GA } & \text { gestational age } \\ \text { IQR } & \text { interquartile range } \\ \text { NICU } & \text { neonatal intensive care unit } \\ \text { OR } & \text { odds ratio } \\ \text { RDS } & \text { respiratory distress syndrome } \\ \text { SGA } & \text { small for gestational age }\end{array}$

\section{References}

1. Engle WA. Surfactant-replacement therapy for respiratory distress in the preterm and term neonate. Pediatrics. 2008; 121:419-32. [PubMed: 18245434]

2. Trembath AN, Clark RH, Bloom BT, Smith PB, Bose C, Laughon M. Trends in surfactant use in the United States: changes in clinical practice. E J Neonatology Res. 2011; 1:23-30.

3. Ramanathan R, Rasmussen MR, Gerstmann DR, Finer N, Sekar K. A randomized, multicenter masked comparison trial of poractant alfa (Curosurf) versus beractant (Survanta) in the treatment of respiratory distress syndrome in preterm infants. J Perinatol. 2004; 21:109-19.

4. Bloom BT, Clark RH. Comparison of Infasurf (calfactant) and Survanta (beractant) in the prevention and treatment of respiratory distress syndrome. Pediatrics. 2005; 116:392-9. [PubMed: 16061594]

5. Bloom BT, Kattwinkel J, Hall RT, Delmore PM, Egan EA, Trout JR, et al. Comparison of Infasurf (calf lung surfactant extract) to Survanta (Beractant) in the treatment and prevention of respiratory distress syndrome. Pediatrics. 1997; 100:31-8. [PubMed: 9200357]

6. Hudak ML, Martin DJ, Egan EA, Matteson EJ, Cummings NJ, Jung AL, et al. A multicenter randomized masked comparison trial of synthetic surfactant versus calf lung surfactant extract in the prevention of neonatal respiratory distress syndrome. Pediatrics. 1997; 100:39-50. [PubMed: 9200358]

7. Ramanathan R, Bhatia JJ, Sekar K, Ernst FR. Mortality in preterm infants with respiratory distress syndrome treated with poractant alfa, calfactant, or beractant: a retrospective study. J Perinatol. 2013; 33:119-25. [PubMed: 21886094]

8. Ramanathan R, Bhatia JJ, Sekar K, Ernst FR. Response to Cummings. J Perinatol. 2013; 33:162-5. [PubMed: 22343394]

9. Singh N, Hawley K, Viswanathan K. Efficacy of porcine versus bovine surfactants for preterm newborns with respiratory distress syndrome: a systematic review and metanalysis. Pediatrics. 2011; 128:1588-95. 
10. Kendig JW, Notter RH, Cox C, Reubens LJ, Davis JM, Maniscalco WM, et al. A comparison of surfactant as immediate prophylaxis and as rescue therapy in newborns of less than 30 weeks' gestation. N Engl J Med. 1991; 324:865-71. [PubMed: 2000109]

11. Ramanathan R. Animal-derived surfactants: where are we? The evidence from randomized, controlled clinical trials. J Perinatol. 2009; 29:S38-43. [PubMed: 19399008]

12. Soll RF. Multiple versus single dose natural surfactant extract for severe neonatal respiratory distress syndrome. Cochrane Database Syst Rev. 2000:CD000141. [PubMed: 10796305]

13. Finer NN, Carlo WA, Walsh MC, Rich W, Gantz MG, Laptook AR, et al. Early CPAP versus surfactant in extremely preterm infants. N Engl J Med. 2010; 362:1970-9. [PubMed: 20472939]

14. Horbar JD, Badger GJ, Carpenter JH, Fanaroff AA, Kilpatrick S, LaCorte M, et al. Trends in mortality and morbidity for very low birth weight infants, 1991-1999. Pediatrics. 2002; 110:14351. [PubMed: 12093960]

15. Rich WD, Leone T, Finer NN. Delivery room intervention: improving the outcome. Clin Perinatol. 2010; 37:189-202. [PubMed: 20363455]

16. Van Marter LJ, Allred EN, Pagano M, Sanocka U, Parad R, Moore M, et al. Do clinical markers of barotrauma and oxygen toxicity explain interhospital variation in rates of chronic lung disease? The Neonatology Committee for the Developmental Network. Pediatrics. 2000; 105:1194-1201. [PubMed: 10835057]

17. Vermont Oxford Network Nightingale Database. Burlington, VT: Vermont Oxford Network; 2012.

18. Laughon MM, Langer JC, Bose CL, Smith PB, Ambalavanan N, Kennedy KA, et al. Prediction of bronchopulmonary dysplasia by postnatal age in extremely premature infants. Am J Respir Crit Care Med. 2011; 183:1715-22. [PubMed: 21471086]

19. Speer CP, Gefeller O, Groneck P, Laufkötter E, Roll C, Hanssler L, et al. Randomised clinical trial of two treatment regimens of natural surfactant preparations in neonatal respiratory distress syndrome. Arch Dis Child Fetal Neonatal Ed. 1995; 72:F8-13. [PubMed: 7743295]

20. Ferrara TB. Effects of surfactant therapy on outcome of infants with birth weights of 600 to 750 grams. J Pediatr. 1991; 119:455-7. [PubMed: 1880661]

21. Horbar JD, Soll RF, Schachinger H, Kewitz G, Versmold HT, Lindner W, et al. A European multicenter randomized controlled trial of single dose surfactant therapy for idiopathic respiratory distress syndrome. Eur J Pediatr. 1990; 149:416-23. [PubMed: 2185026]

22. Cummings JJ. Is there evidence for a mortality difference between natural surfactants? J Perinatol. 2013; 33:161-2. [PubMed: 22343393]

23. Egan EA. In response to mortality in preterm infants with respiratory distress syndrome treated with poractant alfa, calfactant, or beractant: a retrospective study. J Perinatol. 2013; 33:165-6. [PubMed: 23361501]

24. Ramanathan T, Bhatia JJ, Sekar K, Ernst FR. Response to Dr. Egan's letter. J Perinatol. 2013; 33:166-7. [PubMed: 23361502]

25. Walsh M, Laptook A, Kazzi SN, Engle WA, Yao Q, Rasmussen M, et al. A cluster-randomized trial of benchmarking and multimodal quality improvement to improve rates of survival free of bronchopulmonary dysplasia for infants with birth weights of less than 1250 grams. Pediatrics. 2007; 119:876-90. [PubMed: 17473087]

26. Smith PB, Ambalavanan N, Li L, Cotton CM, Laughon M, Walsh MC, et al. Approach to infants born at 22 to 24 weeks' gestation: relationship to outcomes of more-mature infants. Pediatrics. 2012; 129:1508-16.

27. Sturmer T, Jonsson Funk M, Poole C, Brookhart MA. Nonexperimental comparative effectiveness research using linked healthcare databases. Epidemiology. 2011; 22:298-301. [PubMed: 21464649]

\section{Appendix}

Members of the Best Pharmaceuticals for Children Act—Pediatric Trials Network include: The PTN Administrative Core Committee 
Duke Clinical Research Institute, Durham, North Carolina: Daniel K. Benjamin Jr., and Katherine Berezny, Michael Cohen-Wolkowiez, P. Brian Smith; Children's Hospital of Philadelphia, Philadelphia, Pennsylvania: A Jeffrey Barrett and Kelly Wade; University of California-San Diego, San Diego, California: Edmund Capparelli; Children's Mercy Hospital, Kansas City, Missouri: Gregory L. Kearns; University of North Carolina at Chapel Hill, Chapel Hill, North Carolina: Matthew Laughon; Virginia Tech Carilion School of Medicine, Roanoke, Virginia: A Andre Muelenaer; Wake Forest Baptist Medical Center, Winston Salem, North Carolina: T. Michael O'Shea; Penn State College of Medicine, Hershey, Pennsylvania: A Ian M. Paul; George Washington University School of Medicine and Health, Washington, DC: John van den Anker.

Eunice Kennedy Shriver National Institute of Child Health and Human Development

David Siegel, Perdita Taylor-Zapata, Anne Zajicek, Katerina Tsilou, Alice Pagan.

The EMMES Corporation (Data Coordinating Center)

Ravinder Anand, Diane Brandt, Traci Clemons, Gina Simone. 


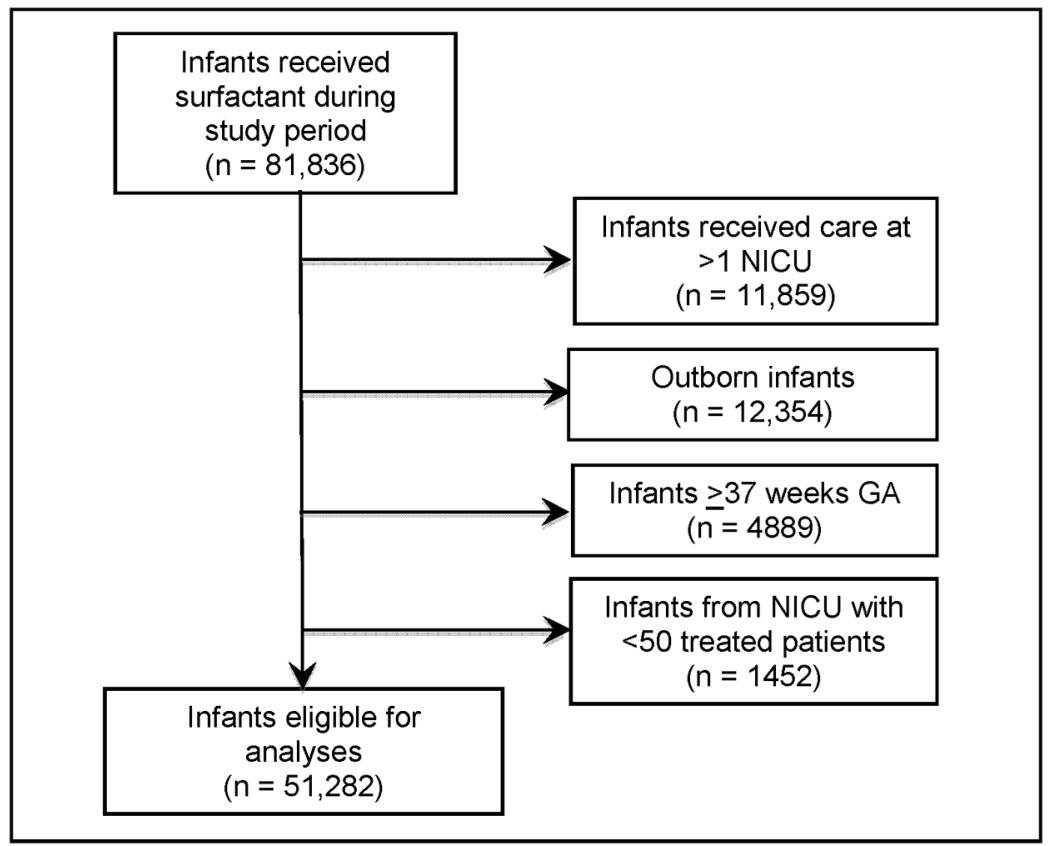

Figure 1.

Flow diagram of study eligibility. Abbreviations: GA, gestational age; NICU, neonatal intensive care unit. 
